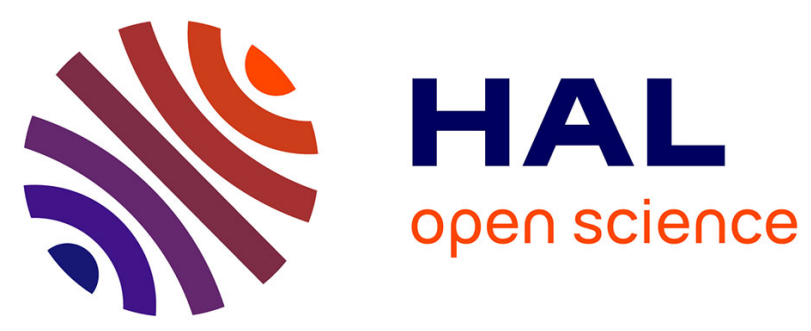

\title{
Unsupervised reconstruction of sea surface currents from AIS maritime traffic data using learnable variational models
}

Simon Benaïchouche, Clement Le Goff, Yann Guichoux, François Rousseau, Ronan Fablet

\section{To cite this version:}

Simon Benaïchouche, Clement Le Goff, Yann Guichoux, François Rousseau, Ronan Fablet. Unsupervised reconstruction of sea surface currents from AIS maritime traffic data using learnable variational models. ICASSP 2021: IEEE International Conference on Acoustics, Speech and Signal Processing, Jun 2021, Toronto, Canada. 10.1109/ICASSP39728.2021.9415038 . hal-03139066

\section{HAL Id: hal-03139066 https://hal.science/hal-03139066}

Submitted on 25 Mar 2021

HAL is a multi-disciplinary open access archive for the deposit and dissemination of scientific research documents, whether they are published or not. The documents may come from teaching and research institutions in France or abroad, or from public or private research centers.
L'archive ouverte pluridisciplinaire $\mathbf{H A L}$, est destinée au dépôt et à la diffusion de documents scientifiques de niveau recherche, publiés ou non, émanant des établissements d'enseignement et de recherche français ou étrangers, des laboratoires publics ou privés. 


\title{
UNSUPERVISED RECONSTRUCTION OF SEA SURFACE CURRENTS FROM AIS MARITIME TRAFFIC DATA USING LEARNABLE VARIATIONAL MODELS
}

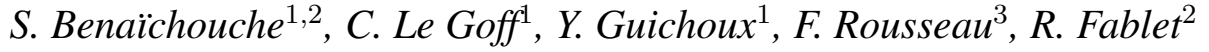 \\ ${ }^{1}$ Eodyn, Brest, FR \\ ${ }^{2}$ IMT Atlantique, UMR CNRS Lab-STICC, Brest, FR \\ ${ }^{3}$ IMT Atlantique, UMR INSERM LaTIM, Brest, FR
}

\begin{abstract}
Space oceanography missions, especially altimeter missions, have considerably improved the observation of sea surface dynamics over the last decades. They can however hardly resolve spatial scales below $\sim 100 \mathrm{~km}$. Meanwhile the AIS (Automatic Identification System) monitoring of the maritime traffic implicitly conveys information on the underlying sea surface currents as the trajectory of ships is affected by the current. Here, we show that an unsupervised variational learning scheme provides new means to elucidate how AIS data streams can be converted into sea surface currents. The proposed scheme relies on a learnable variational framework and relate to variational auto-encoder approach coupled with neural ODE (Ordinary Differential Equation) solving the targeted ill-posed inverse problem. Through numerical experiments on a real AIS dataset, we demonstrate how the proposed scheme could significantly improve the reconstruction of sea surface currents from AIS data compared with state-of-the-art methods, including altimetry-based ones.
\end{abstract}

Index Terms - Sea surface currents, data assimilation, variational learning

\section{INTRODUCTION}

The monitoring of upper ocean dynamics is of key importance for a variety of applications, ranging from maritime traffic routing, oilspill monitoring, marine ecology or climate modeling. This has motivated over the last decades the development of numerous remote sensing technologies dedicated to the reconstruction of sea surface currents, including among others space altimeter missions, HF radar technologies, and SAR imaging. On a global scale, satellite-derived altimeter products are currently the state-of-the-art products for the surface currents. However, they can hardly recover spatial scales below $\sim 100 \mathrm{~km}$ due to the associated scarce space-time sampling.

This work was supported by Eodyn, LEFE program (LEFE MANU project IA-OAC), CNES (grant SWOT-DIEGO) and ANR Projects Melody and OceaniX. It benefited from HPC and GPU resources from Azure (Microsoft EU Ocean awards) and from GENCI-IDRIS (Grant 2020-101030).
As suggested by recent studies [1,2], the worldwide monitoring of the maritime traffic through the Automatic Identification System (AIS) could provide new means to complement the above-mentioned remote sensing technologies. The movement of a ship being not only determined by the considered routing but also affected by sea surface wind and current conditions, one may regard ships as potential samplers of sea surface currents. Given the intrinsic features of AIS data streams, especially the non-linear relationship between the movement of the vessel and the current and the irregular and possibly corrupted space-time sampling of AIS data, solving this inverse problem is a complex issue.

Here, we aim to fully benefit from the available largescale AIS datasets, as AIS data streams involve tens of millions of messages daily on a global scale, and investigate variational deep learning to elucidate sea surface currents from AIS data streams in a weakly supervised fashion. Our key contributions are as follows:

- We introduce a novel physics-informed variational formulation. The physical knowledge is described by a dedicated observation model. The regularization term is defined in a latent space where the unknown underlying geophysical dynamics are modeled using neural ODE [3] prior;

- We show that we can learn the proposed variational model directly from the AIS datasets ;

- We demonstrate the relevance of the proposed approach for a real case-study off South Africa, which comprises 1.32 millions of AIS messages over a 80-day period. We report a relative gain greater than $30 \%$ in terms of reconstruction performance w.r.t. state-of-the-art methods.

The remainder is organized as follows. Section 2 briefly reviews existing studies on AIS-derived sea surface currents, especially the underlying observation model. We describe the proposed learning-based approach in Section 3. We report experiments on a real case-study in Section 4 and Section 5 provides concluding remarks. 


\section{PROBLEM STATEMENT AND RELATED WORK}

AIS is an automatic tracking system required since 2004 by the IMO (International Monitoring Organisation) for security. A typical AIS message contains speed over ground and position information (both from GPS sensors), which may be complemented by a true heading information. By nature, AIS data streams involve an irregular space-time sampling and may be corrupted, which make their direct exploitation in learning schemes challenging [4].

AIS data are mainly used for the surveillance of the maritime traffic [5, 4]. As the sea surface state (i.e. wind, current and wave conditions) affects ship motion, a few studies have also investigated how AIS data might inform sea surface currents [2, 1]. In [1], handcrafted heuristics have been calibrated to convert AIS data into sea surface currents. Though promising, they may lack some physical background to guarantee their generalization capabilities.

In this work, we consider the following linear relationship between sea surface current $U(p, t)$ at a given ship location $p$ at time $t$ and the information conveyed in the AIS message:

$$
\begin{aligned}
S_{\text {ground }}(t) & =L\left(U(p, t), S_{\text {surf }}(t), H(t)\right) \\
& =S_{\text {surf }}(t) H(t)+U(p, t)
\end{aligned}
$$

where $S_{\text {ground }}(t)$ denotes the speed over ground, $S_{\text {surf }}(t)$ refers to the surface speed of the boat, $H(t) \in S^{1}$ denotes a vector pointing towards the known heading direction of the ship. This is an ill-posed problem since one cannot invert this relationship for each position along a trajectory. However, one may benefit from its space-time regularity to recover space-time field $U$ given a dataset of AIS messages over a given area and time period.

In geoscience, space-time inverse problems are classically stated as data assimilation problems. They resort to the minimization of variational costs, which combine an observation model and a prior onto the considered dynamics. Formally, this typically leads to solving

$$
X^{*}=\arg \min _{X} J(X, Y)+R(X)
$$

where $J$ is the data fidelity term, $Y(t)=\left(S_{\text {ground }}(t), H(t)\right)$ is the observation data, $X(p, t)=\left(S_{\text {surf }}(t), U(p, t)\right)$ are the unknown variables to estimate and $R$ denotes the regularization term. The later states the considered prior on the unknown $X$. Optimal Interpolation (OI) [6,7], which is applied for the reconstruction of sea surface currents from satellitederived altimetry data, is an example of such a formulation, where the observation model is linear quadratic and the prior is Gaussian.

A key issue is the design of the regularization term $R($.$) .$ Covariance-based prior considered in OI settings $[6,7]$ are known to poorly represent fine-scale processes. Besides, upper ocean dynamics are governed by complex Navier-Stokes equations and the derivation of simplified representations of sea surface dynamics, e.g. [8], may only represent specific dynamical models. These statements make particularly appealing the recent development of learning schemes for variational models [9], especially to learn a regularization term $R($.$) adapted to the considered observation dataset. We de-$ tail this approach for the retrieval of sea surface currents from AIS data in the next Section. To our knowledge, this is among the first demonstration of the relevance of this approach to sea surface current estimation.

\section{PROPOSED APPROACH}

This Section presents the proposed learning-based variational formulation for the reconstruction of sea surface currents from AIS data. For a given area, let us denote by $Y$ the available set of AIS messages over a time period $T(t \in[0, T])$ and $U$ the spatio-temporal sea surface current field to be reconstructed. Each time period $\Delta_{t}$ typically refers to 8 successive days.

We assume that representative sets of realistic examples of $U$, denoted by $V$, such as numerical simulations or reanalysis products may also be available. Within the variational setting described by Equation (2), we state the reconstruction of fields $U$ from observation data $Y$ as the following minimization

$$
X^{*}, \Theta^{*}=\arg \min _{X, \Theta} J(X, Y)+\lambda_{U} R_{\Theta}(U)+\lambda_{V} R_{\Theta}^{\star}(V)
$$

where $R_{\Theta}$ and $R_{\Theta}^{\star}$ are priors with trainable parameters $\Theta$ to be defined in the next Section. $\lambda_{U}$ and $\lambda_{V}$ are weight parameters balancing the regularization terms w.r.t. the data fidelity term. It has to be noticed that our purpose is to jointly minimize the above variational cost w.r.t. unknown states $U$ and model parameters $\Theta$.

\subsection{Observation term $J$}

Given the observation model defined by Equation (1), we define the observation term $J$ in (3) on the considered spatiotemporal domain $\Omega$ as follows. Given that AIS data are provided as time series of geospatial sparse data merged into consecutive tensors, it is first required to reformulate the observation model w.r.t. the space-time grid considered for spatiotemporal fields. $U \in \mathbb{R}^{\left(T / \Delta_{t}\right) \times N_{x} N_{y} \times 2}$ is a spatio-temporal grid containing current component to estimate, where $T$ is the final time (typically 80 days), $\Delta_{t}$ is the time step ( 8 days) and $\left(N_{x}, N_{y}\right)$ refer to spatial dimensions.

Given these notations, the observation cost $J$ is defined in the following way:

$$
J(X, Y)=\sum_{t=0}^{T}\left\|L\left(U(t), S_{\text {surf }}(t), H(t)\right)-S_{\text {ground }}(t)\right\|_{1, \Omega_{t}}
$$


where $\Omega_{t}$ is the observable spatial domain at time $t$. We may point out that we consider a $L_{1}$ norm in the observation model to account for possibly noisy and/or corrupted AIS messages.

\subsection{Space-time dynamics modeling}

In this work, we investigate the use of a learnable spatiotemporal regularizaton term $R_{\Theta}$ in the considered variational inversion. Given the sparse space-time sampling of AIS data, we expect the regularization term to strongly encode the space-time dynamics. More precisely, we assume the considered space-time dynamics can be well approximated by the solution of an ODE in a low-dimensional manifold. Let us denote by $Z$ the low-dimensional latent state. The considered state-space formulation is as follows:

$$
\left\{\begin{aligned}
U(., t) & =\Phi(Z(t)) \\
Z^{\prime}(t) & =f(Z(t)) \\
Z(0) & =Z_{0}
\end{aligned}\right.
$$

where $f$ is a learnable ODE operator which governs the latent dynamics, $\Phi$ the mapping from the latent space to the space spanned by state $U$ and $Z_{0}$ the unknown initial condition of latent state. Besides, following [10], mapping operator $\Phi$ only depends on a few components of $Z(t)$ to ensure the fact that the dynamics of geophysical field is governed by a non-autonomous ODE.

We may point out that compared with previous works [3, $11,10]$, we address here the identification of this latent ODE representation, when the observation $Y$ is not a direct observation of state $U$ and is sparsely sampled in space and time.

\subsection{End-to-end learning}

Given an AIS dataset for a given area and time period, we aim at jointly learning the considered regularization prior and reconstructing the unknown sea surface currents $U$. Based on the considered latent ODE representations introduced above, the latter comes to estimate the initial conditions for time windows of interest. We also constrain the inversion for successive time windows to be consistent. The end-to-end learning then comes to solve the Equation 3, i.e. finding good estimates of $U$ and spatio-temporal data driven regularization priors.

Inspired by variational auto-encoder framework[12], we propose to make use of a spatial regularization term defined as follows:

$$
R_{\Theta}^{\star}(U)=\sum_{t=0}^{T} K L\left(\Psi\left(U_{t}\right) \| \mathcal{N}(0,1)\right)+\left\|U_{t}-\Phi\left(\Psi\left(U_{t}\right)\right)\right\|_{2}
$$

where $\Psi$ is a learnable operator from spatio-temporal space to the latent space, $K L\left(\Psi\left(U_{t}\right) \| \mathcal{N}(0,1)\right)$ is the KullbackLeibler divergence between a zero-mean and unit-variance normal distribution and the marginal distribution of latent states and $\left\|U_{t}-\Phi\left(\Psi\left(U_{t}\right)\right)\right\|_{2}$ is a reconstruction-based autoencoder loss. To further take into account the dynamics of the sea surface currents, we propose to add in the regularization a penalty loss related to the state-space formulation:

$$
R_{\Theta}(U)=R_{\Theta}^{\star}(U)+\sum_{t=0}^{T-\Delta_{t}}\left\|R K I(Z(t), f)-Z\left(t+\Delta_{t}\right)\right\|_{2}
$$

where $R K I$ is a Runge-Kutta integrator constraining the dynamics of the latent variable $Z$ in time.

Given the scarcity of the sampling of AIS data as well as the noise patterns observed in AIS data streams, we may further constrain the learning step through the use of additional datasets, especially numerical simulations or reanalyses. Though model outputs, including assimilated ones, often depict string mismatch with observation data, they can be regarded as representative examples for the underlying dynamics. This is similar in spirit to energy-based GAN [13]. The overall regularization is then defined as the weighted sum of a spatio-temporal regularization $R_{\Theta}(U)$ and a spatial regularization $R_{\Theta}^{\star}(V)$ on the external example datasets.

\section{APPLICATION TO A REAL AIS DATASET}

\subsection{Experimental setting}

We demonstrate the relevance of the proposed framework through an application to a real raw AIS dataset. We consider a case-study area off South Africa. This area depicts strong and complex sea surface dynamics which are not well resolved by satellite-derived altimetry [14]. Our dataset comprises more than 1 million AIS messages for a 80-day time period from January 1st 2016. Fig.1 provides an illustration of the daily sampling pattern of the AIS data. We also consider GLORYS reanalysis product [15] as a complementary dataset of numerical outputs $V$ which may be used during the training phase as described above.

For performance evaluation, we also collect a dataset of in 682 situ measurements provided by 80 drifters. They provide independent measurements to evaluate the quality of the reconstructed fields in terms of mean square error. For benchmarking purposes, we consider the state-of-the-art altimetry product, referred to $\mathrm{AVISO}^{1}$, and an AIS-derived product combining expert-based processing and optimal interpolation [1].

Regarding the proposed framework, the auto-encoder architecture for the latent representation is as follows: a succession of four convolution layers with ReLu activation followed by a 60-dimensional dense layer for the encoder, a succession of four ConvTranspose layers with ReLU activation except

\footnotetext{
${ }^{1} \mathrm{We}$ refer the reader to the following link for additional information on altimeter-derived AVISO sea surface currents. https://www.aviso.altimetry.fr/en/data/products/sea-surface-heightproducts.html
} 


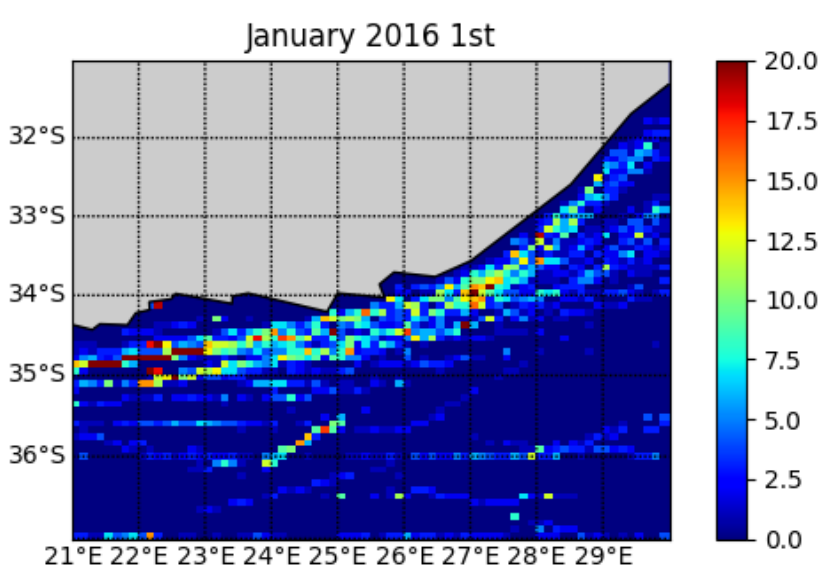

Fig. 1. Example of daily coverage of the AIS data available on a daily basis, here January 16 st 2016, for the considered case-study area, off South Africa.

for the last one where we consider and tanh activation for the decoder, i.e. operator $\Phi$. As mentionned previously, operator $\Phi$ only exploits some components of latent state $Z$, here the ten first. Regarding the ODE operator, we implement a network with 5 dense layers with Soft ReLu activations.

\subsection{Results}

We report in Tab.1 a comparison of the performance of the proposed framework, referred to as VAE-NODE, w.r.t. two baselines. We train the proposed framework either using solely AIS data or using both AIS data and GLORYS data. The former leads to much poorer reconstruction performance, whereas the latter clearly outperforms both the AIS-derived baseline and altimetry-derived one with a relative improvement greater than $40 \%$ in mean square error. We provide an illustration of the reconstructed velocity fields in Fig.2. As expected, the retrieved sea surface current depicts information at finer scales (e.g., the width of the current coming from the North-East of the area) and sharper gradients compared with the altimeter-derived product. Whereas the AIS-derived baseline involves local artifacts, which relate to noise patterns of the AIS dataset and local outliers for simplified observation model (1), the proposed framework retrieves more consistent space-time dynamics. This is regarded as a direct outcome of the considered regularisation through the proposed latent ODE representation. When using AIS and GLORYs data in the training phase, we retrieve much larger velocity values along the high-current area. As the two configurations reach similar values for training loss $J$, this suggests that the additional use of GLORYS data greatly improves the interpolation capabilities of the trainable latent ODE representation.
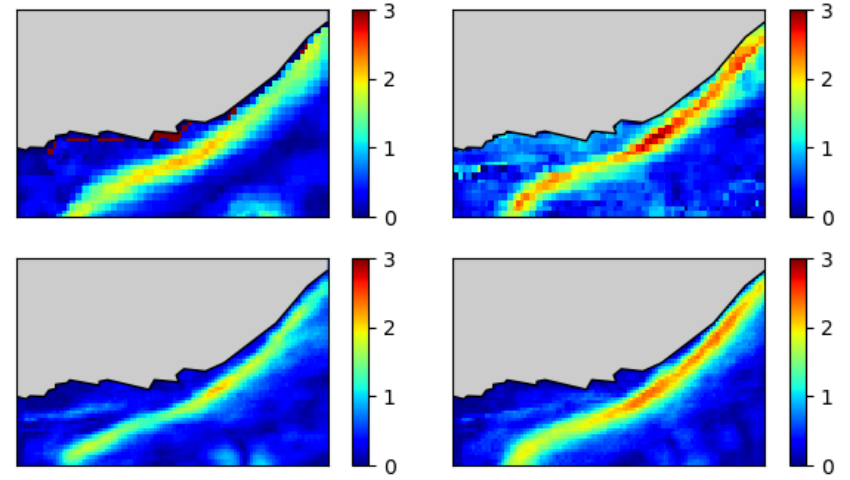

Fig. 2. Reconstructed velocity fields for January 16th 2016 for the altimetry (top, right) and AIS baselines (top left) and the two configurations of the proposed framework with (bottom right) and without (bottom left) the use of GLORYS data in the training phase.

Table 1. Reconstruction performance evaluate from independent in situ data

\begin{tabular}{lll}
\hline \hline Data & Method & MSE \\
\hline \hline Satellite altimetry & OI & 0.1833 \\
\hline AIS & OI & 0.1815 \\
\hline AIS & VAE-NODE & 0.2508 \\
\hline AIS + GLORYS & VAE-NODE & $\mathbf{0 . 1 0 9 8}$ \\
\hline \hline
\end{tabular}

\section{CONCLUSION}

This paper addresses the reconstruction of sea surface current from AIS maritime traffic data. We show that deep learning framework provides new means to address complex inverse problems with irregularly-sampled and noisy real data. We combine a latent ODE representation with a variational formulation. It allows us to exploit some physical knowledge, explicitly through the considered observation model and implicitly through the exploitation of numerical outputs during the training phase.

These results support the relevance of AIS-derived geophysical products to complement satellite-derived ones. Future work will further investigate the evaluation of the proposed framework on other case-studies as well as complementary methodological developments, including data fusion issues for instance to combine altimetry and AIS data.

\section{REFERENCES}

[1] Nicolas Thomas Yann Guichoux, Marc Lennon, "Sea surface current calculation using vessel tracking datas," Maritime Knowledge Discovery and Anomaly Detection Workshop, 2015, e-Odyn. 
[2] Daisuke Inazu, Tsuyoshi Ikeya, Takuji Waseda, Toshiyuki Hibiya, and Yoshinori Shigihara, "Measuring offshore tsunami currents using ship navigation records," Progress in Earth and Planetary Science, vol. 5, no. 1, pp. 38, Aug. 2018.

[3] Ricky T. Q. Chen, Yulia Rubanova, Jesse Bettencourt, and David Duvenaud, "Neural Ordinary Differential Equations," arXiv:1806.07366 [cs, stat], Dec. 2019, arXiv: 1806.07366.

[4] Duong Nguyen, Rodolphe Vadaine, Guillaume Hajduch, René Garello, and Ronan Fablet, "A Multi-task Deep Learning Architecture for Maritime Surveillance using AIS Data Streams," 2018 IEEE 5th International Conference on Data Science and Advanced Analytics (DSAA), pp. 331-340, Oct. 2018, arXiv: 1806.03972.

[5] Fabio Mazzarella, Virginia Fernandez Arguedas, and Michele Vespe, "Knowledge-based vessel position prediction using historical AIS data," Oct. 2015.

[6] Nadal F. Ducet N. Le Traon, P. Y., ," Journal of atmospheric and oceanic technology, vol. 15(2), 1998.

[7] George P. Cressman, "AN OPERATIONAL OBJECTIVE ANALYSIS SYSTEM,” Mon. Wea. Rev., vol. 87, no. 10, pp. 367-374, Oct. 1959, Publisher: American Meteorological Society.

[8] Stephen T. Garner Isaac M. Held, Raymond T. Pierrehumbert and Kyle L. Swanson, "Surface quasigeostrophic dynamics," J. Fluid Mech, vol. 282, pp. 1-20, July 1995.

[9] Ronan Fablet, Lucas Drumetz, and Francois Rousseau, "Joint learning of variational representations and solvers for inverse problems with partially-observed data," arXiv:2006.03653 [cs, eess, stat], June 2020, arXiv: 2006.03653.

[10] Said Ouala, Duong Nguyen, Lucas Drumetz, Bertrand Chapron, Ananda Pascual, Fabrice Collard, Lucile Gaultier, and Ronan Fablet, "Learning Latent Dynamics for Partially-Observed Chaotic Systems," arXiv:1907.02452 [cs, stat], July 2019, arXiv: 1907.02452.

[11] "Discovering governing equations from data by sparse identification of nonlinear dynamical systems | PNAS,"

[12] Diederik P. Kingma and M. Welling, "Auto-encoding variational bayes," CoRR, vol. abs/1312.6114, 2014.

[13] M. Mathieu J. Zhao and Y. LeCun, "Energy-based generative adversarial network," arXiv, 2016.
[14] L. M. Beal Bryden, H. L. and L. M. Duncan, "Structure and transport of the agulhas current and its temporal variability," Journal of Oceanography, vol. 61(3), pp. 479-492, 2005.

[15] N. Ferry, L. Parent, G. Garric, B. Barnier, and N. Jourdain, "Mercator Global Eddy Permitting Ocean Reanalysis GLORYS1V1: Description and Results," 2010. 